УДК 510.643

\title{
Unification and Inference Rules in the Multi-modal Logic of Knowledge and Linear Time LTK
}

\author{
Stepan I. Bashmakov* \\ Institute of Mathematics and Computer Science \\ Siberian Federal University \\ Svobodny, 79, Krasnoyarsk, 660041
}

Russia

Received 10.12.2015, received in revised form 10.01.2016, accepted 15.02.2016

\begin{abstract}
We study unification of formulas in multi-modal LTK logic and give a syntactic description of all formulas which are non-unificable in this logic. Passive inference rules are considered, it is shown that in LTK logic there is a finite basis for passive rules.

Keywords: unification, modal temporal logic, passive inference rules.

DOI: 10.17516/1997-1397-2016-9-2-149-157.
\end{abstract}

\section{Introduction}

The research of unification for various logic systems is one of the most rapidly developing areas of modern mathematical logic. Arisen in the field of Computer Science, primarily in the form of a question about the possibility to transform two different terms in syntactically equivalent by replacing the variables of certain other terms $([1,2])$, from the time the task has changed course on the study of semantic equivalence $([3,4])$.

For most of the non-classical logics (modal, pseudo-boolean, temporal, etc.), there are special dual equational theories of special algebraic systems, so their problems are reduced to the corresponding logical-unificational counterparts ( [5-7]). Basic unificational problem can be viewed as a complex issue: whether the formula is to be transformed into a theorem after replacing the variables (keeping the same values of the coefficients parameters). This issue was investigated and partly resolved (including V. V. Rybakov [8-10]), for intuitionistic and modal logics S4 and Gr $z$.

Unification in intuitionistic logic and in propositional modal logic over the K4 investigated by S. Ghilardi [11-15] (with applications of projective algebra ideas and technology based on projective formulas). In these works the problem of constructing the finite complete sets of unifiers was solved for the considered logic, efficient algorithms were found. Such an approach proved to be a a useful and effective in dealing with the admissibility and the basis of admissible rules (Jerabek [16-18], Iemhoff, Metcalfe [19,20]). Indeed, the existence of computable finite sets of unifiers follows directly solution of the admissibility problem.

Temporal logic is also very dynamic area of mathematical logic and computer science (including Gabbay и Hodkinson [21-23]). In particular, LTL (linear temporal logic) has a significant application in the field of Computer Science (Manna, Pnueli [24,25], Vardi [26,27]). Solving the problem of admissibility of rules in the LTL was proposed by Rybakov [28], basis of admissible

\footnotetext{
*krauder@mail.ru

(c) Siberian Federal University. All rights reserved
} 
rules in LTL by Babenyshev and Rybakov in [29] (without the operator Until [30]). Solution of the unificational problem for LTL has also been found by Rybakov [31,32] and proposed for basic modal and intuitionistic logic in [33,34]. Particularly, in [31] It proved that not all unified in LTL formula are projective, and in [32] proved the projectivity of any unified formula in $L T L_{u}$ (it is a fragment of LTL, only with the operator Until). In the paper of Dzik and Wojtylak [35] they obtained the same result for $S 4.3$.

Research conducted in the present paper, primarily based on the approach proposed in [36]. The key focus here is on the description of non-unifiable formulas in a wide class of modal logics. Especially, it proposed the criteria of non-unifiable (with the proofs) for modal extensions of S4 (Theorem 1.4 below) and $[K 4+\square \perp \equiv \perp]$ (Theorem 1.5). The aim of this article is to investigate the question of unification in linear temporal logic (LTK).

\section{Fundamental definitions and notations}

Before describing the main results, we introduce the most important definitions and notations. Proofs for the most of propositions, consequences and the theorems in this section are detailed in $[36]$.

First, we define a unified formula in this logic. Let $\lambda$ is a logic with the formula $\phi(p, q)$ which describes the equivalent formula. We say that $\alpha$ is equivalent to $\beta$ in $\lambda$,and we write $\alpha \equiv_{\lambda} \beta$ if $\vdash_{\lambda} \phi(\alpha, \beta)$. For convenience, $\phi(\alpha, \beta)$ we denote $\alpha \equiv \beta$.

Definition 1.1. Formula $\alpha\left(p_{1}, \ldots, p_{n}\right)$ is unifiable in an algebraic logic $\lambda$ iff there is a tuple of formulas $\delta_{1}, \ldots, \delta_{n}$ such that $\vdash_{\lambda} \alpha\left(\delta_{1}, \ldots, \delta_{n}\right)$.

Definition 1.2. Formulas $\alpha\left(p_{1}, \ldots, p_{n}\right)$ and $\beta\left(p_{1}, \ldots, p_{n}\right)$ are said to be unifiable in algebraic logic $\lambda$ iff there is a tuple of formulas $\delta_{1}, \ldots, \delta_{n}$ such that $\vdash_{\lambda} \alpha\left(\delta_{1}, \ldots, \delta_{n}\right) \equiv \beta\left(\delta_{1}, \ldots, \delta_{n}\right)$. In this case, the tuple $\delta_{1}, \ldots, \delta_{n}$ is called an unifier for these two formulas.

Corollary 1.3 (2.7 from [36]). For all logics $S I L, S 4_{\text {ext }}, K 4+\square \perp \equiv \perp$ unifiers for formulas can be effectively found among sequences of formulas $\top$ end $\perp$.

For example, by setting $\top$ everywhere in the performance of the variable $p$ and $\perp$ otherwise.

Theorem 1.4 (2.10 from [36]). For any modal logic $\lambda$ extending $S_{4}$ and any modal formula $\alpha$, $\alpha$ is not unifiable in $\lambda$ iff the formula $\square \alpha \rightarrow\left[\bigvee_{p \in \operatorname{Var}(\alpha)} \diamond p \wedge \diamond \neg p\right]$ if provable in $\lambda$.

Theorem 1.5 (2.11 from [36]). For any modal logic $\lambda$ extending $K 4$, where $\square \perp \equiv \perp \in \lambda$ and any modal formula $\alpha, \alpha$ is not unifiable at $\lambda$ iff formula $\square \alpha \wedge \alpha \rightarrow\left[\bigvee_{p \in \operatorname{Var}(\alpha)} \diamond p \wedge \diamond \neg p\right]$ is provable at $\lambda$.

Definition 1.6. Rule $r:=A / B$ is a consequence of the rules $r_{1}:=A_{1} / B_{1}, \ldots, r_{n}:=A_{n} / B_{n}$ in logic $L \Leftrightarrow \forall A \in \operatorname{Var}(L)=\{A \mid A \vDash(\alpha=\top), \forall \alpha \in L\}$ : if

$$
\forall i A \vDash\left(\alpha_{i}=\top\right) \Rightarrow\left(\beta_{i}=\top\right),
$$

then

$$
A \vDash(\alpha=\top) \Rightarrow(\beta=\top) .
$$

Let us recall the definition of algebra formulas, Lindenbaum algebra. Let For is the set of all formulas in the language of logic. We will use the following notation: $A \equiv B \Leftrightarrow(A \rightarrow B) \wedge(B \rightarrow A)$. We write $A \equiv_{L} B$, if $A \equiv B \in L$. Suppose that the logic $L$ has theorem of replacing equivalent. 
Namely, if $\oplus$ is any binary logic connective (for example $\rightarrow, \wedge, \vee$ ), and $A_{1}, A_{2}, B_{1}$ и $B_{2}$ are the formulas, then

$$
\left(A_{1} \equiv_{L} B_{1}, A_{2} \equiv_{L} B_{2}\right) \Rightarrow A_{1} \oplus A_{2} \equiv_{L} B_{1} \oplus B_{2},
$$

and if $\otimes$ is any unary logical connective, then

$$
A_{1} \equiv_{L} B_{1} \Rightarrow \otimes A_{1} \equiv_{L} \otimes B_{1}
$$

Lindenbaum algebra $A / \equiv$ has the basic set For $_{\equiv}$, where

$$
\begin{gathered}
\text { For }_{\equiv}:=\left\{[A]_{\equiv} \mid A \in \text { For },[A]_{\equiv}:=\left\{B \mid B \equiv_{L} A\right\}\right\} . \\
{[A]_{\equiv} \oplus[B]_{\equiv}:=[A \oplus B]_{\equiv} .}
\end{gathered}
$$

We define an algebra $A / \equiv$ as follows: $A / \equiv=\left\langle F o r_{\equiv}, \wedge, \vee, \rightarrow, \square\right\rangle$, where

$$
A \equiv_{L} B \Leftrightarrow(A \rightarrow B) \wedge(B \rightarrow A) \in L
$$

Theorem 1.7 (Lindenbaum). For any modal formula $\alpha$, modal logic $L$ and variables $x_{1}, \ldots, x_{n} \in \operatorname{Var}(L): \alpha\left(x_{1}, \ldots, x_{n}\right) \in L \Leftrightarrow \alpha\left(x_{1}, \ldots, x_{n}\right) \equiv \top$ is a truth on $A / \equiv$.

\section{Semantics LTK}

Alphabet of the language $L^{L T K}$ includes a countable set of propositional variables $P:=$ $\left\{p_{1}, \ldots, p_{n}, \ldots\right\}$, braces $($,$) default Boolean operations and a variety of single modal operators$ $\left\{\square_{\leqslant}, \square_{e}, \square_{1}, \ldots, \square_{n}\right\}$. Every propositional variable $p \in P$ is well-formed formulae (wff), and if $A$ is wff, then so are $\square_{\leqslant} A, \square_{e} A, \square_{i} A(i \in I)$. We abbreviate $F m a\left(L^{L T K}\right)$ as a set of all wff in language $L^{L T K}$ (hereinafter referred to the formula will be understood as formula from the set $\left.F m a\left(L^{L T K}\right)\right)$. Logic operations $\diamond_{\leqslant}, \diamond_{e}, \diamond_{i}$ determined through $\square_{\leqslant}, \square_{e}, \square_{i}$ as follows: $\left.\diamond_{\leqslant}=\neg \square_{\leqslant} \neg, \diamond_{e}=\neg \square_{e} \neg, \diamond_{i}=\neg \square_{i}\right\urcorner$. The values of described modal operators are defined as follows: $\square_{\leqslant} A$ : $A$ is a truth at the current time and in any future; $\square_{e} A$ : $A$ is a truth at a given moment of time; $\square_{i} A$ means that $A$ is a truth in all informational points which available to the agent i. Semantics for the language $L^{L T K}$ models linear and discrete stream of the computational process, in which each point in time is associated with a natural number $n$.

Semantically, our logic is defined on the Kripke frames.

Definition 2.1. k-modal Kripke-frame is a tuple $F=\left\langle W_{F}, R_{1}, \ldots, R_{k}\right\rangle$, where $W_{F}$ is a nonempty set of worlds and each $R_{i}$ is some binary relation on $W_{F}$.

Definition 2.2. Let $F=\left\langle W_{F}, R_{1}, \ldots, R_{k}\right\rangle$ is Kripke-frame, and $\forall R_{i} R_{i}$-cluster is a subset $C^{R_{i}} \in W_{F}$ such that $\forall v, z \in C^{R_{i}}: v R_{i} z \& z R_{i} v$ and $\forall z \in W_{F}, \forall v \in C^{R_{i}}:\left(\left(v R_{i} z \& z R_{i} v\right) \Rightarrow z \in\right.$ $\left.C^{R_{i}}\right)$. For any relation $R_{i}, C^{R_{i}}(v)$ is the $R_{i}$-cluster s.t. $v \in C^{R_{i}}$ or cluster, generated by the element $v . R_{i}$-cluster called: degenerate, if it consists of a single $R_{i}$-irreflexive point; simple if it consists of a single $R_{i}$-reflexive point; proper, if it contains at least two $R_{-}$-reflexive points.

Definition 2.3. LTK-frame is a $k+2$-modal Kripke-frame $F=\left\langle W_{F}, R_{1}, \ldots, R_{k}, R_{e}, R_{\leqslant}\right\rangle$, where: a) $W_{F}$ is the disjoint union of non-empty sets $C^{t}, t \in N: W_{F}:=\bigcup_{t \in N} C^{t}$;

b) $R_{1}, \ldots R_{k}$ are some equivalence relations within each cluster $C^{t}$;

c) $R_{e}$ is universal S5-relation of equivalence at any $C^{t} \in W_{F}$ :

$$
\forall w, z \in W_{F}\left(w R_{e} z \Leftrightarrow\left(w \in C^{t}\right) \&\left(z \in C^{t}\right)\right) ;
$$


d) $R_{\leqslant}$is linear, reflexive, transitive binary temporal relation on $W_{F}$, specifying linear order of clusters (simple chain):

$$
\forall v, z \in W_{F}\left(v R_{\leqslant} z \Leftrightarrow \exists i, j \in N\left(\left(v \in C^{i}\right) \&\left(z \in C^{j}\right) \&(i \leqslant j)\right)\right) ;
$$

Also hold the following properties of matching these relations:

1) $w R_{e} z \Leftrightarrow\left(w R_{\leqslant} z\right) \&\left(z R_{\leqslant} w\right)$;

2) $w R_{i} z \Rightarrow w R_{e} z$.

We denote class of all such frames LTK.

Definition 2.4. For two $R_{\leqslant}$-clusters $C^{m}$ and $C^{j}$ notation $C^{m} R_{t} C^{j}$ indicates that $\forall w \in C^{m}, \forall z \in$

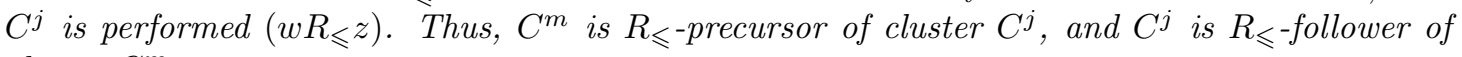
cluster $C^{m}$.

Frames of this class model a situation in which each agent has all the information in the current

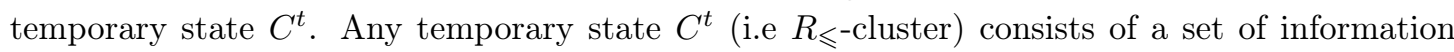
points available at $t$. The relation $R_{\leqslant}$is a connection into a linear stream of information points, wherein for two points $w$ and $z$ term $w R_{\leqslant} z$ means that either $w$ and $z$ are available at the time $t$, or $z$ will be available at subsequent times in relation to $w$. Relation $R_{e}$ connects all information points potentially available at the same moment of time, thus it represents knowledge that is potentially available at any given time. Each relation $R_{i}, i=1, \ldots, n$, reflects the information available to a particular agent $i$.

Definition 2.5. Model $M_{F}$ on a $L T K$-frame $F$ is a tuple $M_{F}=\langle F, V\rangle$, where $V$ is a valuation of a set of propositional letters $p \in P$ on the frame, i.e $\forall p \in P\left[V(p) \subseteq W_{F}\right]$. Given a model $M_{F}=\langle F, V\rangle$, where $F$ is a LTK-frame $W_{F}$. Then $\forall w \in W_{F}$ :

a) $\langle F, w\rangle \Vdash_{V} p \Leftrightarrow w \in V(p)$;

b) $\langle F, w\rangle \Vdash_{V} \square_{\leqslant} A \Leftrightarrow \forall z \in W_{F}\left(w R_{\leqslant} z \Rightarrow\langle F, z\rangle \Vdash_{V} A\right)$;

c) $\langle F, w\rangle \Vdash_{V} \square_{e} A \Leftrightarrow \forall z \in W_{F}\left(w R_{e} z \Rightarrow\langle F, z\rangle \Vdash_{V} A\right)$;

d) $\forall i \in I,\langle F, w\rangle \Vdash_{V} \square_{i} A \Leftrightarrow \forall z \in W_{F}\left(w R_{i} z \Rightarrow\langle F, z\rangle \Vdash_{V} A\right)$.

The relation $\Vdash_{V}$ here means truth relation on the element $w$ of model $M$. Namely, $\langle F, w\rangle \Vdash_{V}$ $A$ means that A true on the element $w$ in model $\langle F, V\rangle$. If the formula A true on any element of the frame $F$ with any valuation $V$, we called A true on the frame $F$ and write $F \Vdash A$.

Definition 2.6. The logic $L T K$ is the set of all LTK-valid formulae on all frames: $L T K:=$ $\left\{A \in F m a\left(L^{L T K}\right) \mid \forall F \in L T K(F \Vdash A)\right\}$. If $A$ belongs to LTK, then we say that $A$ is a theorem of $L T K$.

\section{A criterion of non-unifiability}

We immediately begin with the proof of the main statement of this section.

Theorem 3.1. Any modal formula $A$ is non-unifiable in LTK iff formula

$$
\square_{\leqslant} A \rightarrow\left[\bigvee_{p \in \operatorname{Var}(A)} \diamond_{\leqslant p} \wedge \diamond_{\leqslant} \neg p\right]
$$

is a theorem in $L T K$. 
Proof. 1. Prove the theorem by contradiction. Assume that

$$
\left.\square_{\leqslant} A \rightarrow\left[\bigvee_{p \in \operatorname{Var}(A)} \diamond_{\leqslant} \wedge \diamond_{\leqslant}\right] p\right] \in \operatorname{LTK}
$$

but at the same time, the formula $A$ is unifiable in $L T K$.

Then by definition of unifier, there is a substitution (unifier) $g$ s.t. $g(A) \in L T K$. By the fact that $L T K$ is closed under substitution, we obtain $g\left(\square_{\leqslant} A \rightarrow\left[\bigvee_{p \in \operatorname{Var}(A)} \diamond_{\leqslant p} \diamond_{\leqslant} \neg p\right]\right) \in L T K$.

Let us consider $L T K$-frame $F_{1}$ with all single element clusters (i.e $\forall t: C^{t}=a$ ). Consider the valuation $V$ for all variables $q$ of formulas $g(p)$, where $p \in \operatorname{Var}(A)$, on the $F_{1}: V(q)=\oslash$. Then it is easy to check by the induction on the length of any formula $B$ constructed on variables $q$ that:

$$
\forall b \in F_{1}, \forall c \in F_{1}: b \Vdash_{V} B \Leftrightarrow c \Vdash_{V} B
$$

Consequently,

$$
\forall b \in F_{1}: b \not \Vdash_{V} \bigvee_{p \in \operatorname{Var}(A)} \diamond_{\leqslant} g(p) \wedge \diamond_{\leqslant} g(p)
$$

At the same time,

$$
\forall b \in F_{1}: b \Vdash_{V} \square_{\leqslant} g(A) .
$$

Thereby,

$$
\left.\forall b \in F_{1}: b \not \nvdash_{V} g\left(\square_{\leqslant} A \rightarrow\left[\bigvee_{p \in \operatorname{Var}(A)} \diamond_{\leqslant p} \wedge \diamond_{\leqslant}\right] p\right]\right),
$$

which contradicts the hypothesis:

$$
g\left(\square_{\leqslant} A \rightarrow\left[\bigvee_{p \in \operatorname{Var}(A)} \diamond_{\leqslant p} \wedge \diamond_{\leqslant} p p\right]\right) \in L T K
$$

2. On the contrary, say that the formula $A$ is non-unifiable in $L T K$, but at the same time $\square_{\leqslant} A \rightarrow\left[\bigvee_{p \in \operatorname{Var}(A)} \diamond_{\leqslant p} \wedge \diamond_{\leqslant} \neg\right] \notin L T K$. Then, by finitary approximability of LTK, there is a certain root frame $F$ that disproves this formula:

$$
\exists a \in F:\langle F, a\rangle \mathbb{F}_{V} \square_{\leqslant} A \rightarrow\left[\bigvee_{p \in \operatorname{Var}(A)} \diamond_{\leqslant p \wedge} \diamond_{\leqslant} p p\right] .
$$

That is $\langle F, a\rangle \Vdash_{V} \square_{\leqslant} A$ и $\langle F, a\rangle \not \vdash_{V}\left[\bigvee_{p \in \operatorname{Var}(A)} \diamond_{\leqslant} \wedge \diamond_{\leqslant} \neg p\right]$. Assume this element $a$ as the root of the frame $F_{1}\left(F_{1}=a^{\leqslant}\right)$. By $\langle F, a\rangle \Perp_{V}\left[\bigvee_{p \in \operatorname{Var}(A)} \diamond_{\leqslant} \wedge \diamond_{\leqslant} \neg p\right], \forall p \in \operatorname{Var}(A)$ : either

$$
\text { (1) } \forall b \in F_{1} a R_{\leqslant} b: b \Vdash_{V} p \text {, }
$$

or

(2) $\forall b \in F_{1} a R_{\leqslant} b: b \not_{V} p$.

Choose a substitution $g$ for all of the variables $p$ from the formula $A$ as follows: $\forall p \in \operatorname{Var}(A)$ : $g(p)=\top$ if (1) and $g(p)=\perp$ in the case of (2). Then $g$ is a unifier of the formula $A$. Indeed, if we take any frame $F_{2}$, any cluster $a_{2} \in F_{2}$ and any valuation $V_{2}$ :

$$
a_{2} \Vdash_{V_{2}} A \Leftrightarrow a \Vdash_{V} A .
$$

Therefore, the formula $A$ is unifiable in $L T K$. 


\section{Passive inference rules}

Definition 4.1. Let $r:=A_{1}, \ldots, A_{n} / \beta$ be an inference rule in the logic LTK. The rule $r$ called passive for $L T K$ if for any substitution $g$ of formulas instead of variables in $r$ never $g\left(A_{1}\right) \in L T K \& \ldots \& g\left(A_{n}\right) \in L T K$. In other words $r$ is a passive rule if formulas from its premise have no common unifiers.

Proposition 4.2. For multi-modal logic LTK the rules $r_{n}:=\frac{\bigvee_{1 \leqslant i \leqslant n} \diamond_{\leqslant} p_{i} \wedge \diamond_{\leqslant} \neg p_{i}}{\perp}$ form a basis for all passive rules for $L T K$.

Proof. It is true that $\square_{\leqslant} \bigvee_{1 \leqslant i \leqslant n} \diamond_{\leqslant} \wedge p_{i} \diamond_{\leqslant} \neg p_{i} \rightarrow\left[\bigvee_{1 \leqslant i \leqslant n} \diamond_{\leqslant p} \wedge \diamond_{\leqslant} \neg p\right] \in L T K$, and hence by Theorem 3.1 formula $A_{n}=\bigvee_{1 \leqslant i \leqslant n} \diamond_{\leqslant} \wedge p_{i} \neg p_{i}$ does not unifiable in modal logic $L T K$, i.e any rule $r_{n}$ is passive. Let us assume that a rule $t_{1}:=A_{1}, \ldots, A_{n} / \beta$ is passive for $L T K$. Then the rule $t_{2}:=A_{1} \wedge \cdots \wedge A_{n} / \beta$ is also passive for $L T K$ and formula $A_{1} \wedge \cdots \wedge A_{n}$ is not unifiable in $L T K$. Applying Theorem 4.1 we conclude

$$
\square_{\leqslant}\left(A_{1} \wedge \cdots \wedge A_{n}\right) \rightarrow\left[\bigvee_{p \in \operatorname{Var}\left(A_{1} \wedge \cdots \wedge A_{n}\right)} \diamond_{\leqslant p \wedge} \diamond_{\leqslant} p p\right] \in L T K .
$$

Using the premise of rule $t_{2}$ we conclude

$$
\bigvee_{p \in \operatorname{Var}\left(A_{1} \wedge \cdots \wedge A_{n}\right)} \diamond_{\leqslant p} \diamond_{\leqslant} \neg p
$$

and then applying the rule $r_{n}$, where $n$ is the number of variables in the conjunction of $A_{1} \wedge$ $\cdots \wedge A_{n}$, we can derive the formula $\perp$. From $\perp \rightarrow \beta \in L T K$, in its turn holds $\beta$. Thus, all $r_{n}$ really represent all passive rules in $L T K$.

Now we consider the possibility of reduction infinite (due to an unlimited number of variables) basis of passive rules in $L T K$ that was obtained in the Proposition 4.1 to a finite and more simple form.

Let us remind that the rule $r$ is a consequence of the rules $r_{i} \in X, i \in I$ in a logic $L$, if for any algebra $A \in \operatorname{Var}(L)$ and $\forall i \in I: A \vDash r_{i} \Rightarrow A \vDash r$. Accordingly, a rule $r$ is not a consequence of the rules $r_{i} \in X, i \in I$ otherwise. A rule $r$ true in the algebra $A$ if for any substitution of elements from algebra instead of the variables of a rule $r$ if all formulas from the premise of a rule $r$ is true, then a conclusion formula of $r$ is also true.

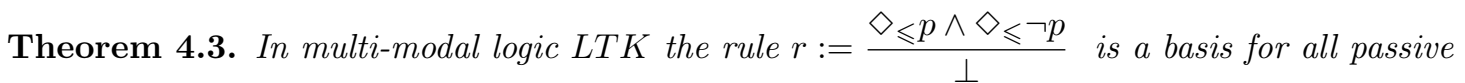
inference rules

Proof. According to Proposition 4.2, it suffices to show that the rules $r_{n}(\forall n)$ are a consequence of $r\left(r \vdash r_{n}, \forall n\right)$.

Suppose that it is not true:

$$
r_{n}:=\frac{\bigvee_{1 \leqslant i \leqslant n} \diamond_{\leqslant} p_{i} \wedge \diamond_{\leqslant} \neg p_{i}}{\perp}
$$

is not a consequence of the rule $r$. Hence there is a finitely generated algebra $\mathrm{A}$, in which the rule $r$ is valid $(A \vDash r)$, but $r_{n}$ is not $\left(A \not \models r_{n}\right)$, thus $\forall i \in(1, \ldots, n)$ there is $a_{i} \in A: \bigvee_{1 \leqslant i \leqslant n} \diamond_{\leqslant} a_{i} \wedge$ $\diamond_{\leqslant} a_{i}=\top$. Get a subalgebra $A_{1}$ of algebra $A$ generated by such elements $a_{i}, 1 \leqslant i \leqslant n$, 
$\left(A_{1}=A_{1}\left(a_{1}, \ldots, a_{n}\right) \subseteq A\right) . A_{1}$ is a $S 4.3$-algebra on $\square_{\leqslant}$. By Lemma 4.3.18 from [10] Kripkeframe $A_{1}^{+}$, associated to $A_{1}$ has a single element reflexive maximal cluster C. By the definition $A_{1}^{+}, \forall a \in A_{1}, a \subseteq A_{1}^{+}$. By hypothesis of proof, $\bigvee_{1 \leqslant i \leqslant n} \vartheta_{\leqslant} a_{i} \wedge \diamond_{\leqslant} \neg a_{i} \in A_{1}$, because $A_{1}$ is a subalgebra $A$, on the construction. Then $\bigvee_{1 \leqslant i \leqslant n} \diamond_{\leqslant} a_{i} \wedge \diamond_{\leqslant} \neg a_{i}=\top=A_{1}^{+}$, but it is impossible on a single element reflexive maximal cluster $\left(C \not \models \bigvee \bigvee_{1 \leqslant i \leqslant n} \diamond \leqslant a_{i} \wedge \diamond \leqslant \neg a_{i}\right)$, and hence $\bigvee_{1 \leqslant i \leqslant n} \diamond_{\leqslant} a_{i} \wedge \diamond_{\leqslant} a_{i} \notin A_{1}^{+}$that contradict with the proof conditions.

\section{References}

[1] A. Robinson, A machine oriented logic based on the resolution principle, J. of the ACM, 12(1965), no. 1, 23-41.

[2] D.Knuth, P.Bendix, Simple Word Problems in Universal Algebras, J.Leech, ed., Pergamon Press, New York, 1970, 263-297.

[3] F.Baader, W.Snyder, Unification theory, In: Robinson J. A., Voronkov A., eds., Handbook of Automated Reasoning. I, Elsevier Science Publishers, 2001, 447-533.

[4] F.Baader, S.Ghilardi, Unification in modal and description logics, Logic Jnl IGPL, 19(2011), no. 6, 705-730.

[5] F.Baader, B.Morawska, Unification in the description logic EL, Logical Methods in Computer Science, 6(2010), 1-31.

[6] F.Baader, P.Narendran, Unification in a description logic with transitive closure of roles, Logic for Programming, Artificial Intelligence, and Reasoning, vol. 2250, LNCS, Springer, 2001, 217-232.

[7] F.Baader, B.Morawska, Unification of concept terms in description logics, Journal of Symbolic Computation, 31(2001), 277-305.

[8] V.V.Rybakov, Problems of Substitution and Admissibility in the Modal System Grz and in Intuitionistic Propositional Calculus, Annals of Pure and Applied Logic, 50(1990), no. 1, 71-106.

[9] V.V.Rybakov, Rules of Inference with Parameters for Intuitionistic logic, J. of Symbolic Logic, 57(1992), no. 3, 912-923.

[10] V.V.Rybakov, Admissible Logical Inference Rules, Series: Studies in Logic and the Foundations of Mathematics, Elsevier Sci. Publ., North-Holland, vol. 136, 1997.

[11] S.Ghilardi, Unification Through Projectivity, J. of Logic and Computation, 7(1997), no.6, $733-752$.

[12] S.Ghilardi, Unification, finite duality and projectivity in varieties of Heyting algebras, $A n$ nals of Pure and Applied Logic, 127(2004), no. 1-3, 99-115.

[13] S.Ghilardi, Unification in Intuitionistic logic, Journal of Symbolic Logic, 64(1999), no.2, 859-880.

[14] S.Ghilardi, Best solving modal equations, Annals of Pure and Applied Logic, 102(2000), 183-198. 
[15] S.Ghilardi, L.Sacchetti, Filtering Unification and Most General Unifiers in Modal Logic, Journal of Symbolic Logic, 69(2004), no. 3, 879-906.

[16] E.Jerabek, Admissible rules of modal logics, Journal of Logic and Computation, 15(2005), 411-431

[17] E.Jerabek, Independent bases of admissible rules, Logic Journal of the IGPL, 16(2008), 249-267.

[18] E.Jerabek, Rules with parameters in modal logic I, CoRR abs/1305.4912, 2013.

[19] R.Iemhof, On the admissible rules of intuitionistic propositional logic, Journal of Symbolic Logic, 66(2001), 281-294.

[20] R.Iemhof, G.Metcalfe, Proof theory for admissible rules, Annals of Pure and Applied Logic, 159(2009), 171-186.

[21] D.M.Gabbay, I.M. Hodkinson, M.A.Reynolds, Temporal Logic: Mathematical Foundations and Computational Aspects, Clarendon Press, Oxford, vol. 1, 1994.

[22] D.M.Gabbay, I.M.Hodkinson, An axiomatisation of the temporal logic with Until and Since over the real numbers, Journal of Logic and Computation, 1(1990), 229-260.

[23] D.M.Gabbay, I.M.Hodkinson, Temporal Logic in Context of Databases. In J. Copeland, editor, Logic and Reality, Essays on the legacy of Arthur Prior, Oxford University Press, 1995.

[24] Z.Manna, A.Pnueli, The Temporal Logic of Reactive and Concurrent Systems: Specification, Springer, vol. 1, 1992.

[25] Z.Manna, A.Pnueli, Temporal Verification of Reactive Systems: Safety, Springer, vol. 1, 1995.

[26] M.Vardi, An automata-theoretic approach to linear temporal logic, Y.Banff Higher Order Workshop, Available at http://citeseer.ist.psu.edu/vardi96automatatheoretic.html, 1995, 238-266.

[27] M.Y.Vardi, Reasoning about the past with two-way automata, Larsen K.G., Skyum S., Winskel G. editors, ICALP, LNCS, Springer, vol. 1443, 1998), 628-641.

[28] V.V.Rybakov, Linear temporal logic with until and next, logical consecutions, Annals of Pure and Applied Logic, 155(2008), 32-45.

[29] S.Babenyshev, V Rybakov, Linear temporal logic LTL: basis for admissible rules, Journal of Logic and Computation, 21(2011), 157-177.

[30] V.V.Rybakov, Logical Consecutions in Discrete Linear Temporal Logic, J. of Symbolic Logic, 70(2005), no. 4, 1137-1149.

[31] V.V.Rybakov, Writing out Unifiers in Linear Temporal Logic, J. Logic Computation, 22(2012), no. 5, 1199-1206.

[32] V.V.Rybakov, Projective formulas and unification in linear temporal logic LTLU, Logic Journal of the IGPL, 22(2014), no. 4, 665-672. 
[33] V.V.Rybakov, Unifiers in transitive modal logics for formulas with coefficients (metavariables), Logic Jnl IGPL, 21(2013), no. 2, 205-215.

[34] V.V.Rybakov, Writing out unifiers for formulas with coefficients in intuitionistic logic, Logic Jnl IGPL, 21(2013), no. 2, 187-198.

[35] W.Dzik, P.Wojtylak, Projective unification in modal logic, Logic Journal of IGPL, 20(2012), no. 1, 121-153.

[36] V.V.Rybakov, M.Terziler, C.Gencer, An essay on unification and inference rules for modal logics, Bulletin of the Section of Logic, 28(1999), no. 3, 145-157.

\section{Унификация и правила вывода в многомодальной логике знания и линейного времени LTK}

Степан И. Башмаков

Институт математики и фундаментальной информатики

Сибирский федеральный университет Свободный, 79, Красноярск, 660041

Россия

$\overline{\text { В статье исследуется унификация формул в многомодальной логике LTK и предложено синтак- }}$ сическое описание всех формул, которые не являются унифицируемыми в данной логике. Рассмотрен вопрос пассивных правил вывода, показано, что в логике LTK есть конечный базис для пассивных правил.

Ключевые слова: унификачия, модальная темпоральная логика, пассивные правила вывода. 